\title{
Subjective Fatigue and Stress Hormone Levels in Urine according to Duration of Shiftwork
}

\author{
Jungsun PARK ${ }^{1}$, Mina $\mathrm{HA}^{2}$, Yunjeong $\mathrm{YI}^{3}$ and Yangho $\mathrm{KIM}^{4}$
}

${ }^{1}$ Department of Safety Management and Policy Research, Occupational Safety and Health Research Institute, Korea Occupational Safety and Health Agency, ${ }^{2}$ College of Medicine, Dankook University, ${ }^{3}$ The Korean Research Institute for Nursing Policy in Korean Nurses Association and ${ }^{4}$ Department of Occupational and Environmental Medicine, Ulsan University Hospital, College of Medicine, University of Ulsan, South Korea

\begin{abstract}
Subjective Fatigue and Stress Hormone Levels in Urine according to Duration of Shiftwork: Jungsun Park, et al. Department of Safety Management and Policy Research, Occupational Safety and Health Research Institute, Korea Occupational Safety and Health Agency, South Korea-To determine the associations between urinary catecholamines and cortisol and subjective complaints of fatigue in shiftworkers, we conducted a field survey of 113 male shiftworkers at a manufacturing company in South Korea from late October to late November in 1999. The shiftwork system in the company was the backward rapidly rotating 4-crew 3-shift, with the morning shift starting at 7:30 AM. Using a self-report questionnaire, we assayed demographic characteristics, past medical history and working hours, as well as subjective complaints of fatigue in 3 categories of questionnaire. We found that, among workers with less than $5 \mathrm{yr}$ of shiftwork experience, the concentrations of norepinephrine, epinephrine (Epi), and dopamine during the afternoon shift were positively correlated with Set II (difficulties in concentration) and Set III (projection of disintegration) of subjective fatigue symptoms. In addition, Epi concentrations were positively correlated with Set I (drowsiness and dullness), II, and III of subjective fatigue symptoms during the night shift. However, among workers with 5 or more years of shiftwork experience, there was no significant positive correlation between urinary catecholamine and subjective fatigue symptoms. In the long term shiftwork experience group, only the complaints scores of Set II fatigue symptoms
\end{abstract}

Received Jul 22, 2005; Accepted Jul 31, 2006

Correspondence to: J. Park, Department of Safety Management and Policy Research, Occupational Safety and Health Research Institute, Korea Occupational Safety and Health Agency, \#34-4 Kusan-dong, Bupyeong-ku, Incheon 403-711, Republic of Korea (e-mail:jsunpark@chol.com) (difficulties in concentration) during the morning shift were significantly higher than those of the short-term shiftwork experience group within the same shift.

(J Occup Health 2006; 48: 446-450)

Key words: Shiftwork, Shiftworker, Catecholamines, Cortisol, Fatigue

Shiftwork systems disrupt circadian rhythms, leading to physical discomfort. It is a major reason for increased accidents among workers due to lowered alertness, as well as psychological anxiety and difficulties in social relationships. For example, Tucker showed that shiftwork affects the state of alertness of employees ${ }^{1)}$. Touitou and Costa found that shiftworkers experienced fatigue, sleep problems, anxiety and gastrointestinal problems ${ }^{2,3)}$, and Ha demonstrated that shiftwork had adverse cardiovascular effects ${ }^{4}$.

Several studies have been conducted on shiftwork and hormone imbalance. For example, Motohashi reported that shiftwork causes disturbances in hormone secretion, leading to disturbances in sleep cycles and physiological variables such as oral temperature and grasping power ${ }^{5}$, and Fujiwara found that shiftwork altered mean heart rate over $4 \mathrm{~h}$ and urinary norepinephrine (NE) concentration ${ }^{6}$. Sluite found that long periods of driving ( $3 \mathrm{~d}$ ) enhanced the urinary excretion of epinephrine (Epi), NE, and

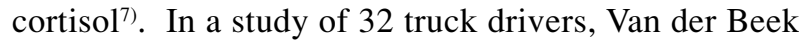
measured Epi and NE in urine samples collected over one working day and one resting day, and found that high physical workload and lack of recovery time led to enhanced excretion of $\mathrm{NE}^{8)}$.

Together, these findings indicate that shiftwork is a stressor that affects physical, psychological and social health. If the subjective fatigue symptoms are positively correlated with excretion of urinary stress hormones positively, we may use fatigue symptom questionnaires 
as a convenient screening tool for finding early detrimental health effects in shiftworkers.

Thus, the purpose of this study was (1) to determine the associations between urinary stress hormones and subjective complaints of fatigue in shiftworkers and (2) in the event of an association, to clarify whether there are the differences in the associations between urinary stress hormones and subjective complaints of fatigue according to the length of shiftwork.

\section{Subjects and Methods}

The subject of this study were employees of a manufacturer of disposable diapers and sanitary items located in Chonan, Chungnam, South Korea. About 70\% of the employees at this factory worked on a 4-crew, 3shift system, which consists of 3 working days, followed by one resting day, and then a switch to another shift. The direction of shift rotation was counter-clockwise, i.e., workers worked the night shift (23:30-07:30), then the afternoon shift (15:30-23:30) and then the morning shift $(07: 30-15: 30)$. All of the workers surveyed were males.

The initial number of subjects was 134 , but we excluded subjects with diseases such as diabetes, hyperthyroidism, hypothyroidism, hypertension, kidney disease, and hyperlipidemia, all of which could affect hormone excretion. Thus, the number of subjects analyzed was 113 . Our research covered 326 shifts, and we failed to gather samples from 13 night shifts.

Meetings were held with the workers of each crew to give presentations about purpose and method of the study, and to encourage them to participate. The volunteers were asked to sign an informed consent statement that was included in the study questionnaire.

\section{Demographic questionnaire}

On the first meeting day, all subjects filled out questionnaires dealing with demographic characteristics, including age, marital status, occupational history before employment by the present company, medical history, drinking characteristics and the major liquor consumed.

\section{Subjective fatigue questionnaire}

The questionnaire for assessing subjective fatigue symptoms was developed by the Japan Society for Occupational Health ${ }^{9)}$. This questionnaire consists of 30 questions addressing 3 sets of symptoms. There are 10 questions in Set I concern drowsiness and dullness. Ten questions in Set II concern difficulties in concentration, and 10 questions in Set III concerning projection of disintegration.

The questionnaires were completed during working hours on the 2 nd or 3rd day of each shift.

\section{Stress hormones in urine}

Urine samples were collected over a period of 2 working hours to measure stress hormones including NE, Epi, dopamine (DA), and creatinine. That is, each subject had an individual appointment to visit the laboratory. Each was asked to urinate $2 \mathrm{~h}$ prior to the appointment, and not to urinate again until visiting the laboratory, when the accumulated urine was collected. The concentrations of NE, Epi, DA, and creatinine were measured by HPLC with an ECD detector. The concentrations of NE, Epi, and DA were adjusted relative to the concentration of creatinine.

In addition, a urine stick test was performed to measure the excretion of occult blood, urinary proteins, and urinary sugars, and in this manner patients with kidney problems were identified and excluded from the analysis.

\section{Data analysis}

The mean shiftwork period was $1,856 \mathrm{~d}$ (range 163 to $3,484 \mathrm{~d}$ ), or about $5.2 \mathrm{yr}$ for the 113 workers. Since $5 \mathrm{yr}$ of shiftwork has been found to be the time at which an individual experiences chronic maladaptation syndrome due to shift-work ${ }^{10)}$, we divided the subjects into two groups: one group $(53.1 \%)$ with less than $5 \mathrm{yr}$ of shiftwork experience (short-term) and the second $(46.9 \%)$ with 5 or more years of shiftwork experience (long-term).

We conducted descriptive analysis to show the difference of the general characteristics of the study subjects and to get medians and ranges of subjective fatigue symptoms, and means and standard deviations of the adjusted hormone levels with creatinine. We also conducted the Mann-Whitney U test to confirm differences in subjective fatigue symptoms, and Student's $t$ test to evaluate the differences in hormone levels according to the length of shiftwork experience. Besides, we calculated correlation coefficients between urinary hormone levels adjusted by creatinine and subjective fatigue according to the length of shiftwork experience.

\section{Results}

The differences of the general characteristics of the subjects according to the length of shiftwork experience are presented in Table 1. Married men comprised $56.7 \%$ of workers with short-term experience and $52.8 \%$ of longterm workers. However, there was no significant difference statistically. In the short-term workers, $83.3 \%$ were below $30 \mathrm{yr}$ old and $13.3 \%$ were $30-39 \mathrm{yr}$ old. In the long-term workers, $64.2 \%$ were below $30 \mathrm{yr}$ old and $20.8 \%$ were $30-39$ yr old with no significant difference between the two groups. Also, there were no significant differences of marital status, smoking, and drinking habit between short-term workers and long-term workers.

We found that, among workers with less than $5 \mathrm{yr}$ of shiftwork experience, the concentrations of NE, Epi, and DA during the afternoon shift were positively correlated with Set II (difficulties in concentration) and Set III (projection of disintegration) of subjective fatigue 
Table 1. General characteristics of subjects according to the length of shiftwork experience

\begin{tabular}{llrrrrr}
\hline & $\begin{array}{c}\text { Short-term } \\
(\mathrm{n}=60)\end{array}$ & $\begin{array}{r}\text { Long-term } \\
(\mathrm{n}=53)\end{array}$ & $\begin{array}{c}\text { chi-square statistics } \\
(p \text { value })\end{array}$ \\
\hline Marital & single & 24 & $(40.0)$ & 18 & $(34.0)$ & \\
status & married & 34 & $(56.7)$ & 28 & $(52.8)$ & $3.80(0.150)$ \\
No. (\%) & NA ${ }^{\text {a) }}$ & 2 & $(3.3)$ & 7 & $(13.2)$ & \\
Age & 20s & 50 & $(83.3)$ & 34 & $(64.2)$ & \\
No. $(\%)$ & 30s & 8 & $(13.3)$ & 11 & $(20.8)$ & $3.18(0.200)$ \\
& 40s & 0 & $(0.0)$ & 1 & $(1.9)$ & \\
Smoking & NA & 2 & $(3.3)$ & 7 & $(13.2)$ & \\
No. $(\%)$ & non-smoker & 7 & $(11.0)$ & 7 & $(13.2)$ & \\
& current smoker & 4 & $(6.7)$ & 5 & $(9.4)$ & $1.06(0.590)$ \\
& NA & 47 & $(78.3)$ & 32 & $(60.4)$ & \\
Drinking & never & 2 & $(3.3)$ & 9 & $(17.0)$ & \\
No. $(\%)$ & little & 5 & $(8.3)$ & 2 & $(3.8)$ & \\
& moderate & 20 & $(33.3)$ & 12 & $(22.6)$ & \\
& heavy & 24 & $(40.0)$ & 19 & $(35.8)$ & $2.19(0.540)$ \\
& NA & 9 & $(15.0)$ & 11 & $(20.8)$ & \\
\hline
\end{tabular}

a) $\mathrm{NA}=$ no answer.

Table 2. Correlation between adjusted urinary hormone levels with creatinine and subjective fatigue according to the length of shiftwork experience

\begin{tabular}{|c|c|c|c|c|c|c|c|c|c|c|}
\hline & \multirow[b]{2}{*}{ Hormone } & \multicolumn{3}{|c|}{ Morning } & \multicolumn{3}{|c|}{ Afternoon } & \multicolumn{3}{|c|}{ Night } \\
\hline & & $\mathrm{NE}$ & Epi & $\overline{\mathrm{DA}}$ & $\mathrm{NE}$ & Epi & $\overline{\mathrm{DA}}$ & $\mathrm{NE}$ & Epi & $\overline{\mathrm{DA}}$ \\
\hline \multirow{3}{*}{ Short term } & Subjective fatigue I & -.250 & -.359 & -.293 & .109 & -.039 & .018 & .217 & $\begin{array}{l}.441^{* *} \\
(.006)\end{array}$ & .167 \\
\hline & Subjective fatigue II & -.028 & -.179 & -.041 & $\begin{array}{l}.450 * * \\
(.002)\end{array}$ & $\begin{array}{l}.441 * * \\
(.002)\end{array}$ & $\begin{array}{l}.339^{*} \\
(.023)\end{array}$ & .216 & $\begin{array}{c}.377^{*} \\
(.021)\end{array}$ & .155 \\
\hline & Subjective fatigue III & -.235 & -.327 & -.221 & $\begin{array}{l}.464 * * \\
(.001)\end{array}$ & $\begin{array}{l}.402 * * \\
(.006)\end{array}$ & $\begin{array}{c}.301 * \\
(.044)\end{array}$ & .141 & $\begin{array}{l}.428 * * \\
(.008)\end{array}$ & .201 \\
\hline \multirow{3}{*}{ Long term } & Subjective fatigue I & -.097 & -.163 & .049 & -.077 & .004 & -.042 & -.290 & -.228 & -.095 \\
\hline & Subjective fatigue II & -.303 & -.356 & -.128 & .006 & -.050 & .080 & -.184 & -.190 & -.045 \\
\hline & Subjective fatigue III & -.121 & -.133 & -.152 & -.013 & -.055 & -.102 & -.192 & -.170 & .019 \\
\hline
\end{tabular}

${ }^{*} p<0.05, * * p<0.01$.

symptoms. In addition, Epi concentrations positively correlated with Set I (drowsiness and dullness), II, and III of subjective fatigue symptoms during night shift. However, among workers with 5 or more years of shiftwork experience, there were no significant positive correlations between urinary catecholamine and subjective fatigue symptoms (Table 2).

The differences in subjective fatigue and excretion of urinary hormones between those with less than $5 \mathrm{yr}$ and those with 5 or more years experience of shiftwork within the same shift were as follows. In the long term shiftwork experience group, only the complaints scores of Set II fatigue symptoms (difficulties in concentration) during the morning shift were significantly higher than those of the short-term shiftwork experience group (Table 3).

\section{Discussion}

Most shiftworkers have difficulties in maintaining regular lifestyles. There are alterations in the rhythm of their lives, leading to deleterious effects on their physical, 
Table 3. Differences in subjective fatigue symptoms and hormone levels within the same shift according to the length of shiftwork experience

\begin{tabular}{|c|c|c|c|c|}
\hline & & Short term & Long term & \\
\hline & Shift & Median (range) & Median (range) & $\begin{array}{c}\text { Mann Whitney U } \\
\text { test ( } p \text { value) }\end{array}$ \\
\hline \multirow[t]{3}{*}{ Subjective fatigue I } & Morning & $3(0-10)$ & $4(0-10)$ & 0.202 \\
\hline & Afternoon & $2(0-9)$ & $1(0-10)$ & 0.352 \\
\hline & Night & $1(0-9)$ & $3(0-10)$ & 0.219 \\
\hline \multirow[t]{3}{*}{ Subjective fatigue II } & Morning & $0 \quad(0-9)$ & $1(0-10)$ & 0.017 \\
\hline & Afternoon & $0 \quad(0-8)$ & $0(0-10)$ & 0.260 \\
\hline & Night & $0(0-6)$ & $1(0-10)$ & 0.056 \\
\hline \multirow[t]{4}{*}{ Subjective fatigue III } & Morning & $1(0-5)$ & $1(0-8)$ & 0.611 \\
\hline & Afternoon & $1(0-8)$ & $1 \quad(0-9)$ & 0.684 \\
\hline & Night & $1(0-7)$ & $1(0-9)$ & 0.296 \\
\hline & Shift & Mean \pm S.D. & Mean \pm S.D. & $t$-test ( $p$ value) \\
\hline \multirow{3}{*}{$\begin{array}{l}\mathrm{NE} \\
\text { (adjusted by creatinine) }\end{array}$} & Morning & $0.62 \pm 0.30$ & $0.63 \pm 0.26$ & 0.800 \\
\hline & Afternoon & $0.66 \pm 0.38$ & $0.69 \pm 0.25$ & 0.714 \\
\hline & Night & $0.54 \pm 0.24$ & $0.58 \pm 0.21$ & 0.452 \\
\hline \multirow{3}{*}{$\begin{array}{l}\text { EPi } \\
\text { (adjusted by creatinine) }\end{array}$} & Morning & $0.16 \pm 0.10$ & $0.17 \pm 0.10$ & 0.609 \\
\hline & Afternoon & $0.14 \pm 0.08$ & $0.17 \pm 0.08$ & 0.086 \\
\hline & Night & $0.12 \pm 0.09$ & $0.14 \pm 0.10$ & 0.433 \\
\hline \multirow{3}{*}{$\begin{array}{l}\text { DA } \\
\text { (adjusted by creatinine) }\end{array}$} & Morning & $2.31 \pm 1.17$ & $2.61 \pm 1.27$ & 0.300 \\
\hline & Afternoon & $2.48 \pm 1.12$ & $2.74 \pm 1.09$ & 0.778 \\
\hline & Night & $2.30 \pm 0.80$ & $2.36 \pm 0.88$ & 0.157 \\
\hline
\end{tabular}

mental, and social functioning. Shiftwork causes an imbalance in circadian rhythms by shifting the time period of work, which adversely affects the physical and mental health of workers. These effects vary from relatively subjective and reversible symptoms, such as declines in short-term memory, increases in fatigue and sleep problems and poor appetite, to major problems including increased mortality rates and deleterious effects on workers' digestive and cardiovascular systems. The 3shift, counterclockwise rotation type of shiftwork system has also been found to increase the rates of nervousness and other psychological problems. All of these health problems reduce the total health level of workers, and increase accident rates and absences due to sickness.

In a study of the physical and mental burdens of work on middle-aged workers, Sudo measured the excretion of catecholamine and cortisol, as well as analyzing the results of a self-administered fatigue questionnaire and stress reports ${ }^{11)}$. Sudo found that middle-aged workers had higher NE excretion than younger workers, even on resting days, and that NE excretion during work was more obvious in middle-aged than in younger workers. While these results indicate that middle-aged workers feel more fatigued at work than younger workers, the latter had higher self-reported fatigue \& stress levels, thus showing a disagreement between subjective and objective data. Our study compared groups of workers with less than 5 and 5 or more years of shiftwork experience. We observed that the excretion of catecholamine by long-term shiftworkers was higher than that by short-term shiftworkers, and that long-term shiftworkers had a higher level of complaints in all three sets of fatigue symptoms. Our finding of agreement between objective and subjective data are thus in contrast to the findings of Sudo. Our results, however, are in agreement with those of Smith and Mason, who showed a correlation between age and subjective fatigue ${ }^{12}$.

When we compared subjective fatigue symptoms and excretion of catecholamines (Table 2), we found that workers with less than $5 \mathrm{yr}$ of shiftwork experience showed a number of positive correlations between subjective fatigue symptoms and catecholamine excretion, whereas more experienced workers showed no positive correlations. This suggests that the less experienced workers may have had difficulty in adjusting to the shiftwork system, leading to increased reports of 
subjective fatigue symptoms, whereas the more experienced workers may have been inured to the shiftwork system and made fewer reports of fatigue. However, this is negated by the finding that more experienced workers reported higher levels of subjective fatigue and enhanced hormone excretion. A possible explanation for these findings is that there may be little correlation between subjective fatigue and the time of the hormone excretion. That is, the less experienced workers reported subjective fatigue at a time in accordance with the hormone excretion, but this synchronocityn was not seen in the more experienced workers. For example, Set I of the subjective fatigue symptoms by less experienced workers was highest during the morning shift, intermediate during the afternoon shift, and lowest during the night shift, which correlated with the excretion of Epi. In contrast, the Set I complaints by more experienced workers were highest during the morning shift, intermediate during the night shift, and lowest during the afternoon shift, whereas Epi excretion was higher during the morning shift and during the afternoon shift and lower during night shifts, thus showing no significant correlation. More experienced workers may have become physiologically adapted to the stress of shiftwork, no longer recognizing it as stressful, even though they reported higher levels of subjective fatigue than less experienced workers.

Besides, there were several other discrepancies in the levels of subjective complaints and hormone excretion by shift. These discrepancies between subjective and objective indices, however, are very difficult to interpret. Thus, further studies on shift workers with 10 or more years of experience or shiftworkers in other occupations are required.

\section{References}

1) Tucker P, Smith L, Macdonald I and Folkard S: Shift length as a determinant of retrospective on-shift alertness. Scad J Work Environ Health 24 (Suppl 3),
49-54 (1998)

2) Touitou Y, Motohashi Y and Reinberg A: Effect of shift work on the night-time secretory patterns of melatonin, prolactin, cortisol and testosterone. Eur J Appl Physiol 60, 288-292 (1990)

3) Costa G, Sartori S, Facco P and Apostoli P: Health conditions of bus drivers in a 6 year follow up study. $\mathrm{J}$ Hum Ergol 30, 405-410 (2001)

4) Ha M, Kim J, Park J and Chung HK: Blood pressure and heart rate variability in workers of 8-hour shifts. J Hum Ergol 30, 229-233 (2001)

5) Motohashi Y: Alteration of circadian rhythm in shiftworking ambulance personnel. Monitoring of salivary cortisol rhythm. Ergonomics 35, 1331-1340 (1992)

6) Fujiwara S, Shinkai S, Kurokawa $Y$ and Watanabe T: The acute effects of experimental short-term evening and night shifts on human circadian rhythm: the oral temperature, heart rate, serum cortisol and urinary catecholamines levels. Int Arch Occup Environ Health 63, 409-418 (1992)

7) Sluiter JK, Van der Beek AJ and Gringes-Dresen MH: Work stress and recovery measured by urinary catecholamines an cortisol excretion in long distance coach drivers. Occup Environ Med 55, 407-413 (1998)

8) Van der Beek AJ, Meijman TF, Frings-Dresen MH, Kuiper JI and Kuiper S: Lorry drivers' work stress evaluated by catecholamies excreted in urine. Occup Environ Med 52, 464-469 (1995)

9) Yoshitake H: Methodological study on the inquiry into subjective symptoms of fatigue. J Sci Labour 47, 709802 (1971)

10) Scott A and LaDou J: Shiftwork: Effects onsleep and health with recommendations for medical surveillance and screening. Occupational Medicine: State of the Art Reviews 5, 273-299 (1990)

11) Sudo A: Evaluation of workload in middle-aged steel workers by measuring urinary excretion of catecholamines and cortisol. Sangyo Igaku 33, 475484 (1991) (in Japanese)

12) Smith L, Mason C: Age and the subjective experience of shiftwork. J Hum Ergol (Tokyo) 30, 307-313 (2001) 